\title{
Future Challenges in State of Charge Estimation for Lithium-Ion Batteries
}

\author{
M. Surendar, P. Pradeepa
}

\begin{abstract}
Energy storage system is an Emerging technology in past few decades. The Energy storage system is an important technology for Electric Vehicles, Hybrid Electric Vehicles (EV) and (HVE) and Micro grid system. The Battery Management System (BMS) is need to be control and monitor the various parameter of the battery such as SOC, SOH, C-Rate, E-Rate ,Temperature, RVL, EOL and so on. However, the (SOC) State of Charge is an important estimation for the online control and BMS monitoring. The SOC is the challenging task when online control and BMS monitoring. This various technique or methods available to estimate the SOC and alsoits represents the Elaboration for various methods of SOC estimation and its drawback. Past five years, where the tendency of the Estimation technique has been oriented towards a mixture of probabilistic techniques and some Artificial Intelligence.
\end{abstract}

Keywords: Battery Management System BMS, Battery Model, Energy Storage, Lithium-ion Battery

\section{INTRODUCTION}

The Smart Grid System, Electric Vehicles and Hybrid Electric Vehicles needed High Technology storage System [1]. The [PbAB] Lead Acid Battery is replaced by [LiB] Lithium-Ion Battery and it is preferred as power supply for above mentioned application and it has the following advantages like large capacity of storage, Long life cycle and no memory [2]. However, some special handling required to avoid the deterioration of better performance and prevent the situation that could result in severe damage. The SOC is an important state to easily track Battery to optimize the performance and extend the Lifetime of the Battery [3].The SOC is defined as the ratio of the rate of available capacity of battery to its maximum capacity when it is fully charged, and describe theremaining capacity of the battery in percentage. The Battery Management System [BMS] includes Analytical model and it consists of Hardware and software which continuously monitor the various state and regulation of Battery. It includes , among others, algorithm to determine the more critical state for micro grid application , batteries not only deliver a certain amount of energy during the Peak Load and satisfied the consumer demand but also provide power different Load Cycle. For this reason, it is important to know the maximum power that can be delivered to and from the battery by charging or discharging respectively, with that SOC needed for making decision concerning the operation.

Revised Manuscript Received on October 10, 2020.

* Correspondence Author

M.Surendar*, Assistant Professor, Department of Electrical and Electronics Engineering, CSI College of Engineering, Nilgiris, Tamil Nadu, India. Email: 2020surendarm@gmail.com

Dr. P.Pradeepa, Associate Professor, Department of Electrical and Electronics Engineering, JAIN Deemed to be University, Bangalore, Karnataka, India.

(C) The Authors. Published by Blue Eyes Intelligence Engineering and Sciences Publication (BEIESP). This is an open access article under the CC BY-NC-ND license (http://creativecommons.org/licenses/by-nc-nd/4.0/)
The Battery operation and performance of its state, particularly SOC is done by Battery Model. There are different kinds of approaches to develop the model [4]. These approaches are simultaneously used to develop Battery Model and some others used different approaches, depending on application [5-7]. Most of the approaches consist of Model with an Algorithm to estimate the SOC [89]. It is important to consider that neither models nor methods are able to fulfill the performance required by demanding the applications [10]. To this end , this paper presents a summary of the key proposals in this literature for SOC estimation in recent years. This review can help the designer to avoid discrimination when choosing the approaches to estimate SOC. This paragraph main goal is to show most recent review of SOC Estimation Methods .A book chapter reported [11,21] contains a brief analysis of SOC Estimation Techniques. While this is a 2020 publication, its bibliographic analysis for SOC Technique includes only 27 database references, articles, transactions and conference proceedings published between 2011 and 2017.The other State Of Battery, the focus of [4] was to present 20\% of 2013, 15\% of 2012 and $65 \%$ of 2011 and earlier. This Review will show the various current trend for SOC Estimation Techniques and its description, merits and main drawbacks that were included from 2017 and 2019. Now, the trend is online implementation of SOC Techniques that includes conventional methods, Adaptive filters, Adaptive artificial Intelligence Methods. The main objective of this paper will show the latest trend and technology for SOC Estimation Techniques that reviewed from 2017 and 2019 for choosing the robust and reliable, online method to Estimate SOC of the Battery for EV and HVE application by the way of reducing the complexity.

II.

BATTERY MANAGEMENT SYSTEM (BMS)

A BMS is a device that built with Hardware \& Software that control the various operation, condition of the Battery to prolong its life, ensuring the safety and providing accurate estimation of the different State of the Battery for the Energy Management Modules.BMS has several features to control and monitor the various states of Battery at different Battery Cell , Battery Module and Battery Pack in order to meet reliable and robustness BMS [1]. The Energy Storage capacity of the Battery has decreases over its lifetime. The SOH (State Of Health) is an essential parameter to predict the deterioration of Battery. The Remaining Useful Life (RUL) is the Remaining Time or Load Cycle until the Battery reaches its End of Life (EOL).

Published By:

Blue Eyes Intelligence Engineering

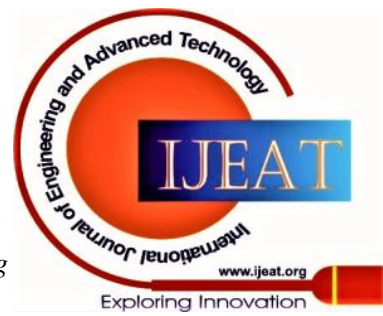




\section{Future Challenges in State of Charge Estimation for Lithium-Ion Batteries}

A BMS must not only have the product of Battery Circuit but also a thorough and accurate device that can estimate SOC, SOH, ROL, EOL and available power to increase the efficiency and safety of the Battery. Fore mentioned parameters can be determined by continuously measuring voltage, current and temperature of the Battery. To estimate SOC is an important key of the Battery but online and accurate estimation remains a challenging task due to NonLinear Dynamic behavior and Continuous Electrochemical Reaction because the Battery Characteristic are continuously changes with its aging[18].The status of the battery charge in a battery management device is like the traditional car fuel meter. The main function of SOC is to communicate with State Of Battery to Driver in order to avoid over charging or discharging [22, 23]. This Literature presents various approaches to design the BMS depends on various function and application. But mostly focusing on a certain function of BMS, such as SOC Estimation [10, 19] or balancing process.

\section{BATTERY MODEL}

The Battery Model is base of the SOC Estimation and various parameter of Battery. It is used to study the Relationship between External Characteristics and Internal States of the Battery. The discrete-time-state-space equations are used in order to estimate the state of charge in different methods.

$$
\begin{aligned}
& X_{K+1}=A_{K} K_{K}+B_{K} U_{K}+W_{K} \rightarrow(1) \\
& Y_{K}=C_{K} X_{K}+D_{K} U_{K}+V_{K} \rightarrow(2)
\end{aligned}
$$

Where, $X_{K}$ denotes $R^{n}$ is the state vector; $V_{K}$ denotes $R^{p}$ is the System input; $W_{K}$ Is the White noise, which can disturb the system. In equation (2); $Y_{K}$ denotes $R^{m} \&$ it is obtained by a Linear Combination of states and input; $V_{K}$ denotes $R^{m}$ is the measurement of Noise and affect the measurement of the system. The Non-Linear compound equation are expressed as equation (3) \& (4);

$$
\begin{aligned}
& X_{K+1}=\mathrm{f}\left(X_{K}, U_{K}\right)+W_{K} \rightarrow(3) \\
& Y_{K}=g\left(X_{K}, U_{K}\right)+V_{K} \rightarrow(4)
\end{aligned}
$$

The system model is developed based on equation from (1) to (4).The system update its State \& Output based on the Input. The equation (1) \& (3) is called "System Equation" in order to determine stability, dynamic controllability and sensitivity to disturbance occurs. The equation (2) \& (4) is known as "Measurement Equation", While matrices $A_{K}, B_{K}, C_{K}, D_{K}$ Describes the system's dynamics which can differ in time depending on the Battery Model technique used. Every particular Battery Model technique prescribes the estimating parameters. That may not be directly Measured Equation (1) \& (2) are illustrated in Figure 1. And equation (3) \& (4) are illustrated in Figure 2.

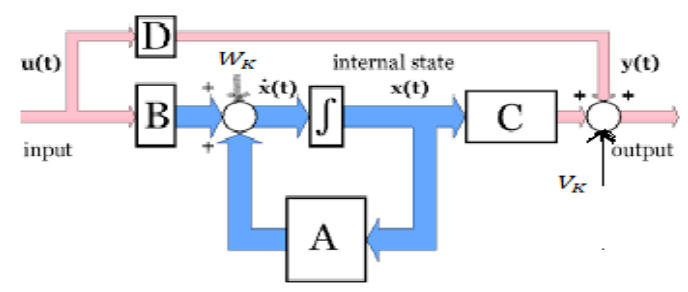

Fig.1 Linear Model

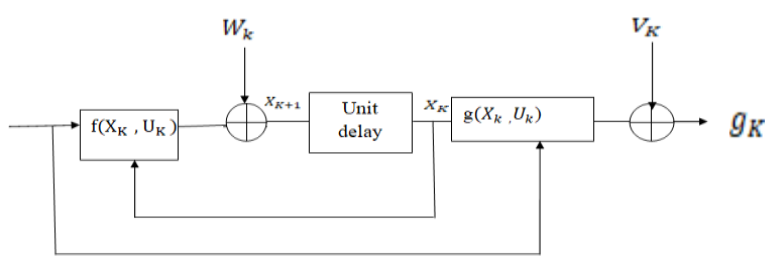

Fig.2 Non-Linear Model

Commonly there are two models such as Electrochemical and Electric Circuit Model. By using equation (1) to (4) these models are prescribed. In Ref[24] Electrochemical Model having some difficulties to estimate the Battery parameter due to some drawback such that high computational complexity to obtain parameter more over special software can be used to estimate the Battery parameter. The (ECM) Electric Circuit Model can avoid these difficulties in order to estimate the Battery parameter. It consists of Open circuit Voltage Source (OCV) , Internal Resistance $\left(\mathrm{R}_{\mathrm{t}}\right)$, parallel combination of diffusion Resistance $\left(R_{p}\right)$ and diffusion Capacitance $\left(C_{p}\right)$ and Terminal Voltage $\left(V_{t}\right)$ for every single cell. A Battery Pack consists of hundreds of single cell then describes the ECM model in each and individual cell having more complexity. In Ref [25] present each and every cell model can be replaced by the Thevenins Equivalent Model from that easily determine the parameters of the Battery. In Ref[26] present the model equation for $n$ cells and these equations are included as a single system by using equation (1) and (2).

\section{METHODS FOR SOC ESTIMATION}

The SOC is defined as the ratio of current remaining capacity to maximum capacity of Battery when fully charged under different condition. SOC Estimation is an important part of BMS.The SOC Estimation methods are classified the various group depends on Theoretical and Experimental characteristics. The Figure 3 shows various classification and can be described three methods such that Direct Methods, Adaptive Filter Method, Adaptive Artificial Intelligence Method and other new methods. Based on innovative ideas, this review will show comparison of these methods and its drawbacks.

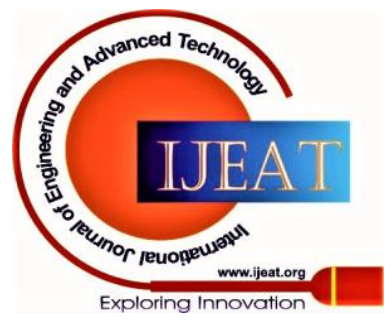




\begin{tabular}{|c|c|c|c|c|}
\hline Direct method & $\begin{array}{c}\text { Adaptive Filter } \\
\text { Method }\end{array}$ & $\begin{array}{c}\text { Adaptive } \\
\text { Artificial } \\
\text { Intelligence }\end{array}$ & $\begin{array}{c}\text { Other methods } \\
\text { based on } \\
\text { Innovation }\end{array}$ \\
\hline Coloumb counting & Kalman Filter & Fuzzy Logic & $\begin{array}{c}\text { Principle } \\
\text { Component } \\
\text { Analysis }\end{array}$ \\
\hline $\begin{array}{c}\text { Open Circuit Voltage } \\
\text { Method }\end{array}$ & $\begin{array}{c}\text { Extended } \\
\text { Kalman Filter }\end{array}$ & $\begin{array}{c}\text { Neural } \\
\text { Networks }\end{array}$ & $\begin{array}{c}\text { Support Linear } \\
\text { Regression }\end{array}$ \\
\hline \hline $\begin{array}{c}\text { Electrochemical } \\
\text { Internal Resistance }\end{array}$ & Least Square & $\begin{array}{c}\text { Fuzzy Neural } \\
\text { System }\end{array}$ & $\begin{array}{c}\text { Combination } \\
\text { Algorithm }\end{array}$ \\
\hline & Square & Genetic & $\begin{array}{c}\text { Algorithm } \\
\text { Algorithm }\end{array}$ \\
\hline & & PSO & $\begin{array}{c}\text { Sequential } \\
\text { MUNTE CARLO }\end{array}$ \\
\hline
\end{tabular}

Fig.3 Methods of SOC

\section{A. DIRECT METHOD}

The Direct Method is conventional method of SOC Estimation which directly measure the physical properties of the Battery such that voltage, current and temperature. By using some equation SOC can be estimated.

\section{B. COLOUMB COUNTING METHOD}

This method is known as Ampere hour Balancing Method and it is simplest technique to estimate the state of charge for short time. It is a most standard method for Industry to Estimate SOC Ref [28]. The SOC can be defined by the equation (6), at any time,

$\operatorname{SOC}(\mathrm{t})=\operatorname{SOC}\left(\mathrm{t}_{0}\right)+\frac{1}{\mathrm{C}_{\mathrm{n}}} \int_{\mathrm{t}_{0}}^{\mathrm{t}_{0+1}}\left(\mathrm{I}_{\mathrm{bat}}\right) \mathrm{d} \tau \times 100(6)$

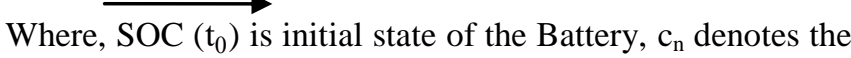
normal capacity of the Battery, $\mathrm{I}_{\text {bat }}$ implies the charging or discharging current. The CC Method is a simple method; it has Initial Error and Accumulated Error. The Battery Current Measurement has Measurement Error and Measurement Noise due to sensors. These Error leads to some Errors in Hole Estimation Process. Hence it is necessary for some supporting Algorithm in order to reduce this Mean Square Error. The initial state is known in equation (5) when measure some quantity from the Battery. By practice, it will assume this lead to errors in Estimation. This method has more sensitive to the initial SOC. While it has been used extensively in recent years, CC is not generally used only as a method to estimate SOC but is often used in conjunction with other techniques Ref [29].The CC method is incorporated with other Adaptive Algorithm in order to reduce the Mean Square Error, during the SOC Estimation few paper presented Ref $[28,29,30]$ Fore mentioned Technique.

\section{OPEN CIRCUIT VOLTAGE METHOD}

OCV Method is known as "Direct Voltage Measurement Method" Ref[21].The SOC Estimation imposes characteristics of SOC and OCV either Direct Voltage Measurement or Model Based Method Ref [31].

The voltage can be calculated continuously in the OCV method and the corresponding SOC is obtained from the table. The OCV Method is inherent difficult in practical situation. It is necessary the sensor have high resolution to measure the voltage accurately and some time required to attain equilibrium. The OCV Method is required, some rest time to measure SOC ultimately it is not used for real time or online application. However, the OCV Method is used for calibrating the CC Method in equation (6) and this method incorporate with other Adaptive Technique Ref $[36,37]$.

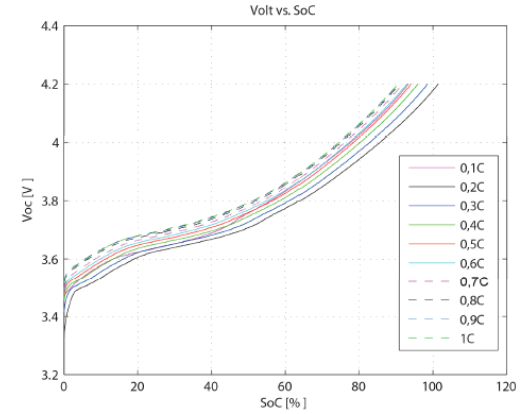

Figure 4. $\mathrm{OCV} v \mathrm{vs}$. SoC during the charge process at different current values.

Figure 4, shows OCV-SOC characteristics for different current Ref [21]. This curve is obtained from experimental data for LiNico $\mathrm{MnO}_{2}$ Battery and it will show, why OCVSOC curve cannot be used directly to estimate SOC for the following reason (i) When a significant amount of current is received, charging hits the upper limit voltage faster than when less current is received.(ii) At the same time, Open OCV, the battery has different SOC values depending on the current it receives, with higher values of SOC when developing charging phase by values of small current. Thus smallest value of Error makes some divergence to SOC Estimation. However, the OCV-SOC curve can incorporate with other methods. In Ref [35] the OCV-SOC curve is used for Electric Circuit Model with an Extended Kalman Filter to Estimate the SOC. In Ref [36] the SOC Estimation method is combined with Open-Circuit Voltage and Kalman Filter Method.

\section{INTERNAL RESISTANCE METHOD}

Internal Resistance method in which estimate the SOC based on, the relationship between Internal Resistance of the battery and SOC. The relationship between Internal Resistance of the Battery and SOC is obtained based on the relationship between Internal Resistance of battery and Voltage change Ref [3] of the Battery Ref[37,38]. Figure 5, shows basic Electric Circuit Model to evaluate the Open Circuit Voltage ,by this equation (8).

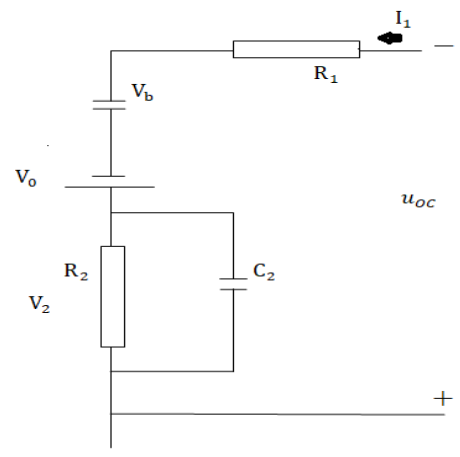

Fig.5 Electric Circuit Model

From that model 5,We apply the KVL, the Open Circuit Voltage estimated, In equation eight $R_{1}$ is the battery Ohmic Resistance; $R_{2}$ is the battery's Polarization Resistance that forms an RC loop parallel to the $\mathrm{C}_{2}$ Polarization Convector. The effect of polarization within the battery can be simulated.

Published By:

Blue Eyes Intelligence Engineering and Sciences Publication

(C) Copyright: All rights reserved.

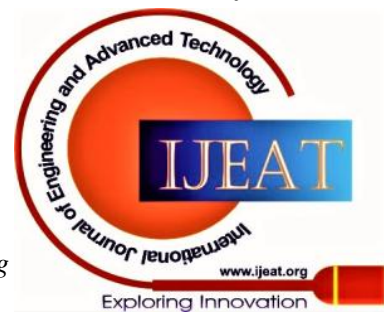

Exploring Innovation 
$\mathrm{C} \mathrm{b}$ is the battery capacitor which is blocked with the current $\mathrm{I}_{1}$ due to the OCV shift.

The Internal Resistance Method is to estimate the SOC during the discharge period in practically. This Method is quitesimple only, because to consider Discharge Current and Internal Resistance of the Battery. However, in practice, the relationship between Battery parameter and SOC quit complex. At initial stage, the efficiency of Internal resistance of the battery is stable and no fluctuation. However, in later stage the internal resistance increases obviously due to change in voltage and current \& more fluctuation .so, Internal Resistance Method can be used in later stage of Discharge.

As all we know, the Internal Resistance can bedivided into two parts, such as AC Internal Impedance and DC Internal Resistance. So, this method has divided into two parts depends on AC or DC. The AC Impedance is a transmitting mechanism between the voltage and the battery current. The AC Impedance Meter is normally calibrated. However, due to the great influence of temperature, it has always controversial whether it should be estimated in the open circuit state or in the process of charging \& discharging. Therefore, this method is rarely used in real Vehicles. In Ref [39,40] the Internal Resistance method is quit complex to SOC estimation for NickelHydrogen and Li-ion Battery than Lead-Acid Battery. But , at present Li-ion Battery were used for EV and HVE Application. Therefore Internal Resistance Method, is not suitable for SOC Estimation to EV and HVE Application. The internal resistance can be estimated from the voltage change-to-current ratio. This approach is not ideal for SOC estimates each time the value is modified. However, the SOC can be determined using another CC process.

\section{ADAPTIVE FILTER METHOD}

Adaptive Filter Method is the combination of Direct and OCV Model Based Method. By using the feedback to Adapt the system to change the current output according to the varying input Ref[13].

\section{A. KALMAN FILTER METHOD}

Adaptive Kalman Filter Theory or Algorithm can be used to estimate SOC of Li-ion Battery.The Electric Circuit Model has incorporate with KF Algorithm to estimate the SOC of the Battery.In ECM model consider Battery Model derive from equation (1) and (2) for which Input such that Current and Temperature and Output has Voltage.

The principle of this method is shown in Figure 6 and 7.It describe the Battery as a system composed of an State Equation (1) and Observed Equation (2). $X_{K+1}=A_{K} K_{K}+$ $B_{K} U_{K}+W_{K}$ and $Y_{K}=C_{K} X_{K}+D_{K} U_{K}+V_{K}$ Consider SOC as an internal state of thee system, establishes a State-Space Model and makes the minimum variance of SOC Estimation.

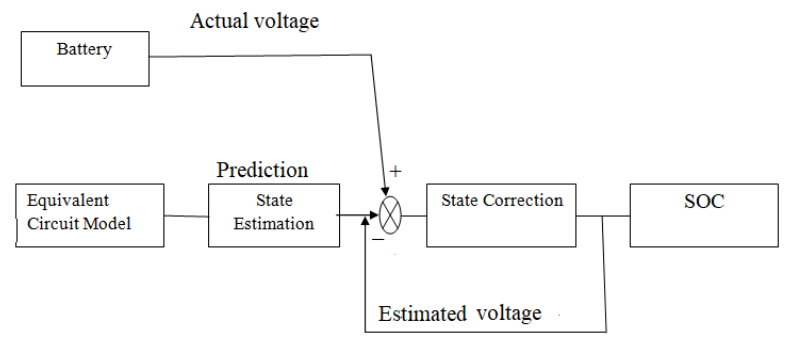

Fig.6 Kalman Filter Process

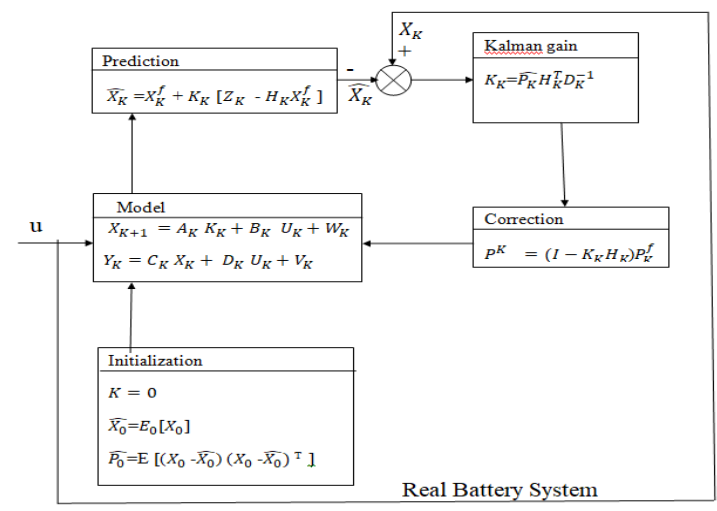

Fig. 7 Kalman Filter Algorithm

Figure 7 describes KF Theory basic equation for estimation of SOC for the various State of Battery Ref[21].The prediction is given .Forecast state and the forecast and observation is given Estimated state of the Battery.

At present, the various method have been generated based on this method Ref [40], SOC Estimation of Lead Acid battery. By combination of CC Algorithm and OCV method with Extended Kalman Filter.However, this method is not suitable for Real time applications such as EV and HVE. The Li-ion Battery is more suitable for mentioned application. At present Ref [32] Adaptive Sigma Kalman Filter Algorithm is replaced by Extended Kalman Filter in order to reduce the computational complexity, uncertainty problem and to improve accuracy where Estimation of SOC is obtained. At present Ref [33] unscented Kalman Filter is replaced by Extended Kalman Filter for Estimation of SOC by improving Linearity, reduce the sampling noise and minimize the mean square error.

The Advantage of this method is to eliminate the Error from CC and OCV Method as well as .If the initial value has a certain deviation it can well converge to the real value. The Disadvantage of this method Ref [22] , the accuracy is largely depends on the ECM Model and the Error is mainly comes from these aspects: the time, variability of the model, the non-linearity of the model and approximate treatment of noise.

\section{B. EXTENDED KALMAN FILTER}

It is advanced method based on Basic Kalman Filter. The solution of non-linearity problem is obtained from this filter. The system model is consider as equation (3) and (4) such that $X_{K+1}=\mathrm{f}\left(X_{K}, U_{K}\right)+W_{K}$ and $Y_{K}=$ $g\left(X_{K}, U_{K}\right)+V_{K}$.

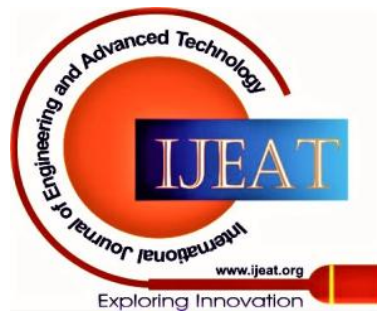


The EKF Filter linearize the Non-linear Problem in order to Estimate the SOC of Battery. At present Ref [31] Basic KF is replaced by EKF , have some shortcoming in accuracy, real time and operability. The EKF Algorithm uses the Error between the model and the true value as a compensation form to correct the predicted value. Figure 8,shows Basic principle of EKF Ref [40].

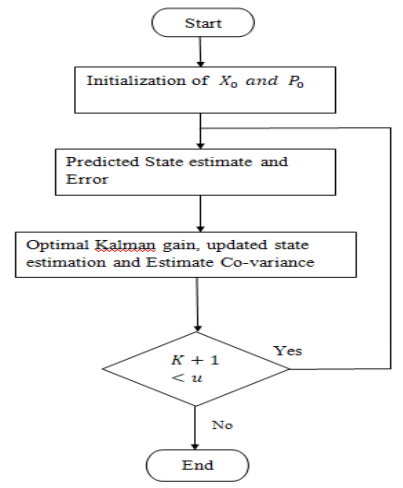

Fig.8 Basic principle of EKF

However, disadvantage of this method is less accuracy that UKF and having more noise both voltage and current signal to compensate these drawbacks. At present Ref [33] the improved Algorithm such that UKF combined with ECM Model to estimate the SOC.

\section{LEAST MEAN SQUARE FILTER}

The Linear LS Estimation is another improved method to estimate the SOC of the Battery in order to reduce the Mean Square error.In LS estimation, the observed data are represented as $X=A \theta+e$ where " $A$ " is assumed as Known Full Rank Matrix; $\theta=\left(\theta_{1}, \theta_{2}, \ldots \theta_{\mathrm{n}}\right)^{\mathrm{T}}$ is a Parameter vector; e denotes Noise in random. The Linear Estimation minimize the Sum Square Error given by $\mathrm{I}(\theta)=$ $(\mathrm{X}-\mathrm{A} \theta)^{\mathrm{T}}(\mathrm{X}-\mathrm{A} \theta)$ as the cost function.The result minimize the Sum Error Square followed by the $A^{T} A \widehat{\theta_{L S}}$ $=A^{T} X$. The LS Estimator is given by $\theta_{L S}=\left(A^{T} A\right)^{-1} A^{T} X=$ $A^{T} X$, where $A^{T}$ is the pseudo inverse of " $A$ ". The matrix inversion makes LS Estimation computationally complex. The LMS Algorithm is computationally simple but has the following drawbacks; (i) LMS convergence slow, particularly when the Eigen value of the autocorrelation matrix $R_{Y}$ widely spread. (ii) The step size parameter has to be chosen properly. (iii) Excess Mean Square Error is high. At present Ref [27], during depth of discharge in EV and HEV application by using LMS Filter is used to estimate SOC of different battery.

\section{RECURSIVE LEAST SQUARE ESTIMATION}

The RLS (Recursive Least Square Estimation) Algorithm is presently used to Estimate the SOC of the Battery. The RLS having advantages then LMS (i) It converges faster when the Eigen values spread of Autocorrelation matrix is large.(ii) The problem of step size parameter is avoided. Figure 9, shows the basic principle of RLS Filter.

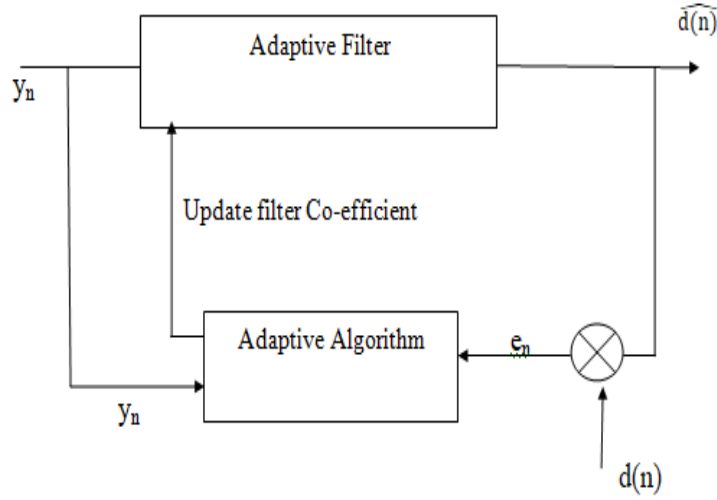

Fig.9 Principle of RLS filter

The LS approach minimize the Sum Square Error between the derived signal $d(n)$ and filtered output $\overline{d(n)}$.It consider all available data up to the current time for determining the filter parameter. The filter is optimum with respect to all the available data in the Least Square sense. The associated LS Estimation problem is solved recursively, Figure 10, shows the Basic RLS Algorithm.

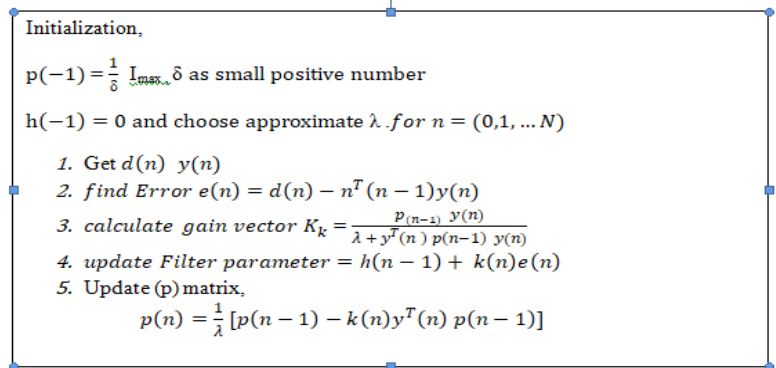

Fig.10 Recurssive least square process

Least Square Method calculates system parameter values that minimize the error between measured output and desired signal by assuming system disturbance such as white noise. RLS Method is used to Adaptive Filters to find the co-efficient that allow the minimum square of the signal.The RLS Algorithm makes this process recursively and updates its estimated parameter value by incorporating the new information from every sampling time.

At present Ref [27], the RLS Algorithm combined with Extended Kalman Filter with ECM Model in order to Estimate the SOC with real time changes for the application of EV and HVE applications.

\section{ADAPTIVE ARTIFICIAL INTELLIGENCE METHOD}

Artificial Intelligence is one of the powerful toolsto estimate the various state of any system. In this method, Model has trained like Human brain to adapt any condition and identify any disturbance meanwhile; it can reduce the error and estimate the state near to the objective function or cost function.

\section{A. ARTIFICIAL NEURAL NETWORKS}

Artificial Neural Networks is one of the Branch of Artificial Intelligence. It is an emerging tool to estimate the SOC of different types of battery. It is based on simple simulation like Human brain and accepts the corresponding training through the input and output sample.

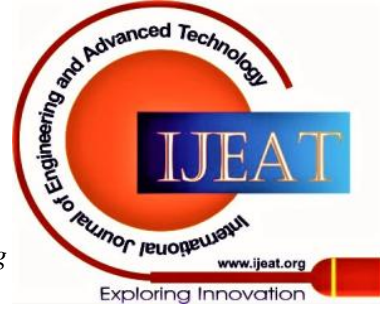


The Neural Network consists of three layer such that input layer, hidden layer and output layer. The Input layer is feed by Battery parameter such as Current, Voltage, Temperature and Internal Resistor and Output layer generate SOC for the particular state. The Hidden layer is an important role for weight updating and bias. This algorithm consists of feed forward pass and back propagation process. Thefeed forward pass has processing the data from input layer to output layer trough hidden layer with bias.So; the Output of Hidden and Output layer $\operatorname{areH}_{\mathrm{n}}=\sum_{\mathrm{i}=1}^{\mathrm{N}} \mathrm{X}_{\mathrm{i}} \mathrm{W}_{\mathrm{i}}+$ $\mathrm{b}_{\mathrm{n}}$ and $\mathrm{y}_{\mathrm{n}}=\sum_{\mathrm{i}=1}^{\mathrm{N}} \mathrm{X}_{\mathrm{i}} \mathrm{W}_{\mathrm{i}}+\mathrm{b}_{\mathrm{n}}$ respectively.

The Sigmoid Activation function in each layer by the following equation, $\square_{\square}=\frac{1}{1+\square^{-\square}}$.In other hand, the Back Propagation process is used to reduce the Error which is close to the objective function by updating the weight between output and input layer. These process continuously run until it reach nearest objective function. In Figure 10 \& 11 shows, the Architecture and process of Neural Network Ref [22].

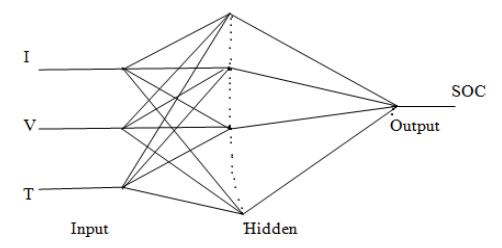

Fig. 10 SOC Estimation principle of Neural Network

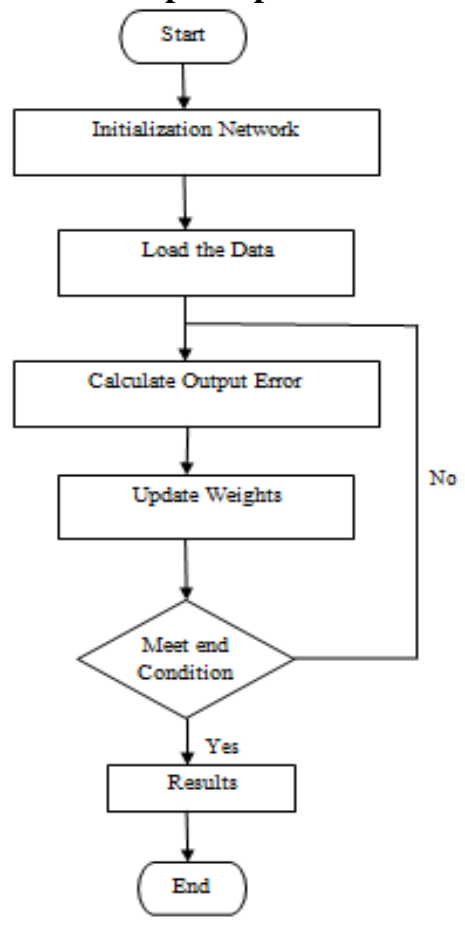

Fig. 11:Process of ANN

In Ref [28] ,The SOC estimated by using the Single Layer Neural Network.The non-measurable parameter such as SOC Estimated from the measurable parameter of Battery such as voltage and current.The ANN trained for experimental data derived from Battery OCV/ SOC Relationship. In Ref [21] ,Describe the Radial Basis Neural Network (RBNN) to estimate the SOC for determining the
Battery.In this paper ,its combination of improved coloumb counting method with the BPNN of SOC Estimation.

The following drawback of ANN is that, it needs more Neurons to increase the Accuracy, which limits the implementation in real time models. In addition, each ANN needs to be trained before it can be used and large iteration required to train it. For that reasona trained ANN can be used for Specific Applications.

\section{FUZZY LOGIC SYSTEM}

Fuzzy Logic System is the Branch of Artificial Intelligence and simplifies noisy, vague and imprecise input data by using Objective base rule.The operation of Fuzzy Logic consist of four simple stage (i) Process Control: Identify the condition (antecedent) and action ( consequent) variable of the process;(ii)Fuzzification: Measurement of input variable are converted into appropriate Fuzzy set to express measurement un certainties;(iii) Fuzzy Inference Engine:It is used to measure the Fuzzy measurement and evaluate the rule stored in the rulebased;(iv)Defuzzification: The fuzzified output from Inference Engine is converted into single crisp value. Fuzzy Logic system estimate the state of system such that (High,Low) rather than Precise number.The Approaches presented [28] ,the Fuzzy Rule Based System is used to estimate the gain of Kalman Filter and genetic Algorithm is used to evolve the definition of Fuzzy Rule Based System.In Ref [29],the venin ECM is combined with Coloumb Counting method is adapted by Fuzzy Logic Control Scheme to estimate the SOC of the Battery. The equalization is based on the State Of Charge (SOC) is proposed in Ref [32]. The venin equivalent circuit model of Lithium ion battery as well as the Extended Kalman Filter Algorithm is employed for SOC Estimation for Effective equalization.The FLC is proposed to reduce the Energy consumption and equalization time.But,this method does not consider battery aging in real time applications.The approaches presented Ref [35],the new Fuzzy Logic Sliding mode observer for SOC Estimation.The second order is a circuit equivalent resistance with capacitor model to explain the charge or discharge output of the battery.. The Fuzzy logic Controller is introduced to improve the performance of sliding mode observed, forming the Fuzzy Logic Sliding Mode observed (FLSMO).

The main drawback of this system has high computational cost,the system is clearly defined as Fuzzy rule based on the Battery characteristics. The proposal methods are not consider the aging effect because the battery parameter can change over the Lifetime of Battery.

\section{B. GENETIC ALGORITHM}

It is a optimization Technique for given problem or population. This technique is used to estimate the SOC of the Battery directly or it is used to evolve the best solution from the Battery parameter Figure 12,shows the basic process of Genetic Algorithm which impose the three basic steps such as population, initialization, crossover, mutation to obtain the best solution or optimized solution. aging effect of Battery.In this paper there is a comparison between convectional NN and RBNN for estimating aging effect on SOC.In Ref [28],present the Noval Back Propagation Method is used to SOC Estimation for the

Published By:

Blue Eyes Intelligence Engineering

and Sciences Publication

(C) Copyright: All rights reserved.

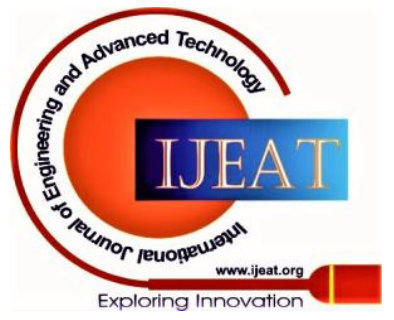

DOI:10.35940/ijeat.A1789.1010120

Journal Website: www.ijeat.org 


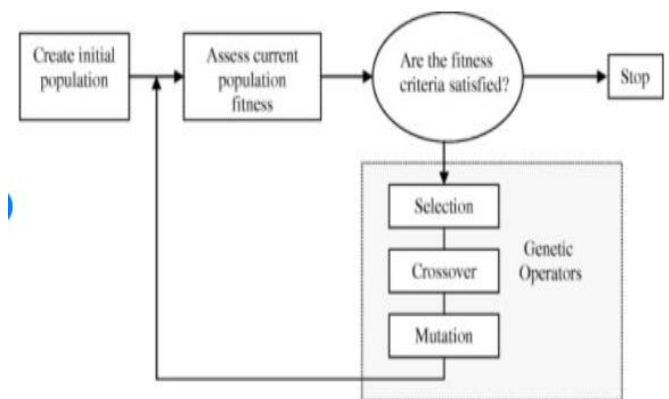

Fig.12 Genetic Operation

Genetic Algorithm is an optimization technique to estimate different state of the Battery.The approaches presented Ref [36,37], Genetic Algorithm technique is used to estimate optimized solution of Battery parameter such that voltage, current and temperature from Electric Circuit Model.In Ref [38],the Genetic Algorithm technique is used to estimate the battery parameter from second order.ECM which depends on SOC.The goal is to find the polynomial coefficient values of parameter equations, which generate an OCVSOC function that best fits.The characteristic curve is obtained from the manufacturer's battery data.In Ref [39], the GA Technique is used to identify the Initial SOC from that we illustrate the various technique. Some drawback in GA based on Estimation technique as follows;(i) It demands heavy computation and delay in optimization response time.(ii) Fine tuning of parameters is required to get effective results.

\section{PARTICLE SWARM OPTIMIZATION}

Particle Swarm optimization technique is one of the intelligent technique to take decision fast for given random population than Genetic Algorithm Ref [21]. This Algorithm consists of a population of particles randomly initialized in the search space.In this process it generates Random population and nextpopulation based on cost function. So, thePSO is fast finding solution than Genetic Algorithm. The approaches was presented Ref $[12,23]$,the PSO method is used to determine the unknown parameter of second order ECM to obtain OCV.The approaches presented Ref[34] ,the PSO Algorithm is used to estimate the second order ECM parameter but SOC is calculated using two different methods;External Kalman Filter and Smooth Variable Structure Filter (SVSF). In Ref [15] present a Statistical Learning Technique for SOC Estimation is known as Multivariate Adaptive Regression Splines (MARS), whose optimal parameters are calculated using PSO.

\section{E. INNOVATIVE IDEAS FOR DIFFERENT METHODS}

There are variety of SOC Estimation Algorithm when we go in depth of research it is found to be SOC algorithm have strong adaptability and high accuracy rate. The most SOC Algorithm follows the best innovative ideas. They are derived from the knowledge of the subject area or it may be a combination of different methods to get the advantage of other methods Ref [18-28]. The important methods used on Least Square Method and Zero Mean Method .The important algorithm is based on Multi-variable statistics best uses the PCA (Principle Component Analysis) which is used to analyze the process data and for comprising and simplifying different data. Anyhow the above said method does not find suitable for different degree of battery deterioration and at different temperature. Based upon the experiment revealed that the factors affecting the SOC performance are current,voltage and resistance and further they used least square methods to deal with the main factors that affects the SOC performance and with the model simulation they test the feasibility for the method.The experimental results reveal they are not applicable for temperature variability but this method effective improvement was shown in PCA. It is also different from degradation of battery. The improvement was also found in Mean Fault Error. The SVR is also commonly known as Vector Regression, which gives the basic idea for model complexity and to minimize the structural empirical risk.By comparing with other methods like Neural Network, it is easy to apply and has good practicability to get better value of SOC. To get the better generalization ability it is very important for Kernel function to be selected in SVR to get a proper Output. In Gaussian radial function method it is generally obtained to get fast convergence speed and a simple non-linear mapping and it is also used to find other parameters.In the case Zhujaing found that SVR Method has good stability and accuracy and prediction rate when compared to Neural Network Algorithm as he made the input as voltage,temperature and output as SOC.In many area of research, the researchers used to combine more algorithms and found use of each algorithm resulting in the more accurate and Adaptive Algorithm.By combining three methods namely An Integral Method and Open Circuit Voltage Method, Load Voltage Method.A new estimation method has been obtained by Deng Tao.et.al.

The OCV method gives the initial value for SOC and the value of SOC $^{-1}$ was obtained by an integration method and the value of load voltage method was averaged by SOC ${ }^{2}$.Based on Neural Network and Master Slave Adaptive Uncentered Kalman Filter Algorithm (MS)-AUKF Algorithm. An SOC Estimation was found by Wuet.al.

Another Researcher Linzin proposed a method with drift an integral method with the noise combination and drift current where the estimation of SOC lithium battery was achieved with the use of CKF. Anyhow continuous improvement will be carried out in estimation methods and updated. The current scenario continuously improves the response speed and accuracy.

\section{CONCLUSION}

Due to its own advantages, Lithium batteries have been very popular among the Researchers to be utilized in the field of Electrical Vehicle which have been in the current trend in the Automobile sectors and it has been very important for the Battery Management System and the Estimation of SOC becomes the vital role. Anyhow the above review discuss the specific improvement in SOC Estimation with different methods and specific research areas and classifies the existing methods for future research. From the above discussion it is very clear that SOC

Published By:

Blue Eyes Intelligence Engineering

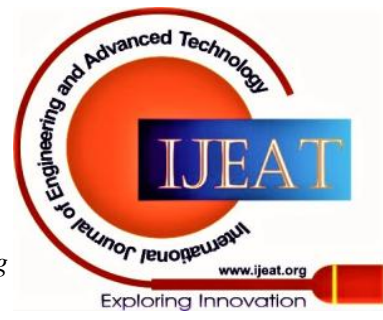


estimation can be done in specific areas which has its own pros and loss the comparison has been made and tabulated which gives the clear idea for the future research in the area of batteries.

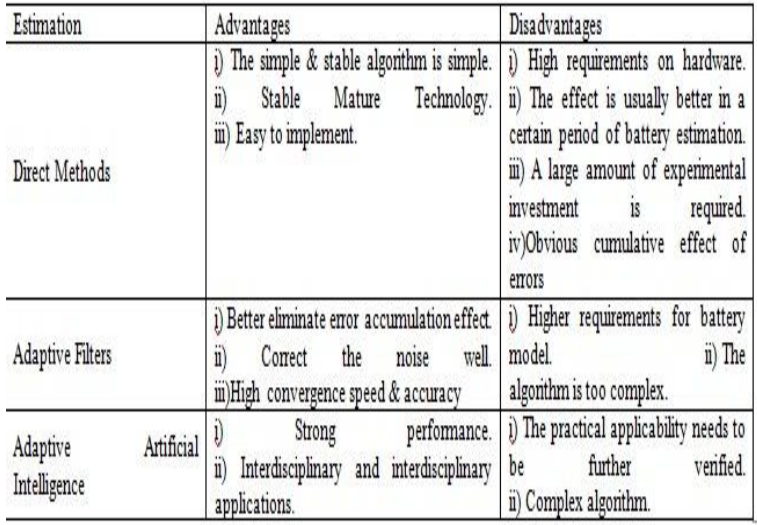

Fig.12 SOC estimation

From the above figure it is very clear that the traditional algorithm is very simple and highly used algorithm for the current SOC estimation methods. But anyhow elimination accuracy has to be improved when compared with modern methods. Modern trend of elimination method have higher accuracy rate where it is limited with hardware technology.

And now-a-days researches uses mostly combined algorithm. Internal resistance methods are not usually used alone. They will have a combination with An-Integral method to have a higher advantage. The accurate estimations of non-linear systems and online estimation of SOC algorithm have been found with KF-series. The KFseries algorithm can improve the SOC estimation accuracy rate with the use of Particle Filter series algorithm. Anyhow the above KF-series deals with high complexity in its operation.The effectively reduced operational complexity can be improved by Trackless Particle Filter algorithm. Neural Network method, Linear Model method and many other methods are still to be improved

\section{CURRENT AND FUTURE DEVELOPMENTS}

Due to the limitation of traditional estimation methods have their own nature of estimation. Nowadays they are often used with combination of other methods and account for highest proportion. The modern methods of estimation using control theory for SOC will have higher response speed and high precision. Obviously combination will lead to complexity in algorithm which requires strong theory for support. The use of the algorithm and combination for the improvement of accuracy and speed for requirements of the industries needs in the higher end. As me trending into the technological most of the innovative ideas have been developed in the laboratical stage much vehicles. Taking into the consideration of SOC estimation methods it is found to be the traditional estimation method will be the basic main stream for the vehicles.To get the extensive promotion in the area of battery research, the SOC estimation should be narrow down by the future researches and there is a need for lot of experiments and advanced hardware facilities to be developed. As in turn the automobile sectors have been moving into the world of more improvements to be done in the area of real time

hybrid vehicles the development in this area specifically will lead to a green global world.

\section{REFERENCES}

1. Antón, J.C.Á.; Nieto, P.J.G.; Gonzalo, E.G.; Pérez, J.C.V.; Vega, M.G.; Viejo, C.B. A new predictive model for the state-of-charge of a high-power lithium-ion cell based on a pso-optimized multivariate adaptive regression spline approach. IEEE Trans. Veh. Technol. 2016, vol.65, pp.4197-4208.

2. Cacciato, M.; Nobile, G.; Scarcella, G.; Scelba, G. Real-time modelbased estimation of soc and soh for energy storage systems. IEEE Trans. Power Electron. 2017, vol.32, pp.794-803.

3. Cai, Y.;Wang, Q.; Qi,W. D-ukf based state of health estimation for 18650 type lithium battery. In Proceedings

4. Chaoui, H.; Golbon, N.; Hmouz, I.; Souissi, R.; Tahar, S. Lyapunovbased adaptive state of charge and state of health estimation for lithium-ion batteries. IEEE Trans. Ind. Electron. 2014, vol.62, pp.1610-1618.

5. Chaoui, H.; Golbon, N.; Hmouz, I.; Souissi, R.; Tahar, S. Lyapunovbased adaptive state of charge and state of health estimation for lithium-ion batteries. IEEE Trans. Ind. Electron. 2014, vol.62, pp.1610-1618.

6. Chaoui, H.; Gualous, H. Adaptive state of charge estimation of lithium-ion batteries with parameter and thermal uncertainties. IEEE Trans. Control Syst. Technol. 2017, vol.25, pp.752-759.

7. Chaoui, H.; Ibe-Ekeocha, C.C.; Gualous, H. Aging prediction and state of charge estimation of a lifepo4 battery using input timedelayed neural networks. Electr. Power Syst. Res. 2017, vol.146, pp.189-197.

8. Chen, J.; Ouyang, Q.; Xu, C.; Su, H. Neural network-based state of charge observer design for lithium-ion batteries. IEEE Trans. Control Syst. Technol. 2017, PP, 1-9.

9. Chen, Z.; Fu, Y.; Mi, C.C. State of charge estimation of lithium-ion batteries in electric drive vehicles using extended kalman filtering IEEE Trans. Veh. Technol. 2013, vol.62, pp.1020-1030.

10. Cuma, M.U.; Koroglu, T. A comprehensive review on estimation strategies used in hybrid and battery electric vehicles. Renew. Sustain. Energy Rev. 2015, vol.42, pp.517-531.

11. Feng, Y.; Cao, Z.; Shen,W.; Yu, X.; Han, F.; Chen, R.;Wu, J. Intelligent battery management for electric and

12. Fleischer, C.;Waag,W.; Bai, Z.; Sauer, D.U. Adaptive on-line state of available power prediction of lithium-ion batteries. J. Power Electron. 2013, vol.13, pp.516-527.

13. Ghantous, D.; Berkowitz, F.; Maluf, N. Method and Circuitry to Calculate the State of Charge of a Battery/Cell.U.S. Patent 8791669B2, 29 July 2014.

14. Hannan, M.A.; Lipu, M.S.H.; Hussain, A.; Mohamed, A. A review of lithium-ion battery state of charge estimation and management system in electric vehicle applications: Challenges and recommendations. Renew. Sustain. Energy Rev. 2017, vol.78, pp. 834-854.

15. Hongwei, L.; Caiying, S. Methods of State of Charge Estimation of Electric Vehicle. Automot. Eng. 2017, pp.31-33.

16. Hongwei, L.; Caiying, S. Methods of State of Charge Estimation of Electric Vehicle. Automot. Eng. 2017, pp.31-33.

17. hybrid electric vehicles: A survey. In Proceedings of the 2016 IEEE International Conference on Industrial Technology (ICIT), Taipei, Taiwan, 14-17 March 2016; pp. 1436-1441.

18. Khayat, N.; Karami, N. Adaptive techniques used for lifetime estimation of lithium-ion batteries. In Proceedings of the 2016 Third International Conference on Electrical, Electronics, Computer Engineering and their Applications (EECEA), Beirut, Lebanon, 2123 April 2016; pp. 98-103.

19. Klass,V.; Behm, M.; Lindbergh, G. Capturing lithium-ion battery dynamics with support vector machine-based battery model. J. Power Sources 2015, 298, pp.92-101.

20. Lillehei, C.W.; Cruz, A.B.; Johnsrude, I.; Sellers, R.D. A new method of assessing the state of charge of implanted cardiac pacemaker batteries. Am. J. Cardiol. 1965, vol.16, pp.717-721.

21. Liu, C.; Liu, W.; Wang, L.; Hu, G.; Ma, L.; Ren, B. A new method of modeling and state of charge estimation of the battery. J. Power Sources 2016, vol. 320, pp.1-12.

Published By:

Blue Eyes Intelligence Engineering and Sciences Publication

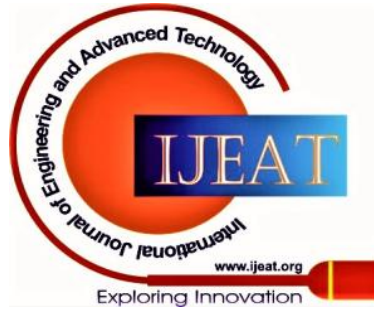

(C) Copyright: All rights reserved. 
22. Lu, L.; Han, X.; Li, J.; Hua, J.; Ouyang, M. A review on the key issues for lithium-ion battery management in electric vehicles. J. Power Sources 2013, vol.226,pp. 272-288.

23. Ng, K.S.; Moo, C.-S.; Chen, Y.-P.; Hsieh, Y.-C. Enhanced coulomb counting method for estimating state-of-charge and state-of-health of lithium-ion batteries. Appl. Energy 2009, vol.86, pp.1506-1511.

24. of the 2016 IEEE International Conference on Mechatronics and Automation, Harbin, China, 7-10 August 2016;pp. 754-758.

25. Peishan, Y.; Yin, B. Analysis of SOC Estimation Algorithm for Electric Vehicle Power Battery. Automot. Pract. Technol. 2019, vol.15, pp.15-17.

26. Peishan, Y.; Yin, B. Analysis of SOC Estimation Algorithm for Electric Vehicle Power Battery. Automot. Pract. Technol. 2019, vol.15, pp.15-17.

27. Ping, S.; Lu, L.; Gao, M.; Dong, R.; Xuning, S.F. Combined Estimation Method for Lithium Ion Battery State of Charge, State of Health and State of Function. CN105301509A, 3 February 2016.

28. Rahimi-Eichi, H.; Ojha, U.; Baronti, F.; Chow, M.Y. Battery management system: An overview of its application in the smart grid and electric vehicles. IEEE Ind. Electron. Mag. 2013, vol.7, pp.4-16.

29. Tang, X.; Liu, B.; Gao, F. State of charge estimation of lifepo4 battery based on a gain-classifier observer. Energy Procedia 2017, vol.105, pp.2071-2076.

30. Tingting, Y.; Jie, Z.; Linkai, Z.; Yuhua, Z. SOC Estimation and Simulation of Lithium Battery Based on Improved Ampere-hour Integral Method. Energy Sav. New Energy 2018, vol.6, pp.58-60.

31. Waag, W.; Fleischer, C.; Sauer, D.U. Critical review of the methods for monitoring of lithium-ion batteries in electric and hybrid vehicles. J. Power Sources 2014, vol.258, pp.321-339.

32. Weng, C.; Sun, J.; Peng, H. A unified open-circuit-voltage model of lithium-ion batteries for state-of-charge estimation and state-ofhealth monitoring. J. Power Sources 2014, vol.258, pp.228-237.

33. Wu, H.-D.; Xiao-ming, R.;Wei, N.; Chao, H. Estimating SOC of Liion battery by improved AH combined with neural network. Battery Bimon. 2016, vol.46, pp.16-19.

34. Wu, Z.; Shang, M.; Shen, D.; Qi, S. SOC estimation for batteries using MS-AUKF and neural network. J. Renew. Sustain. Energy 2019, vol.11, pp.1-10.

35. Xiong, R.; Tian, J.; Mu, H.; Wang, C. A systematic model-based degradation behavior recognition and health monitoring method for lithium-ion batteries. Appl. Energy 201, vol.207,pp.372-383.

36. Yang, Y.; Cui, N.;Wang, C.; Liu, M.; Gao, R. SOC estimation of lithium-ion battery based on new adaptive fading extended Kalman filter. In Proceedings of the 2017 Chinese Automation Congress (CAC), Jinan, China, 20-22 October 2017.

37. Yu, H.; Duan, J.; Du, W.; Xue, S.; Sun, J. China's energy storage industry: Develop status, existing problems and countermeasures. Renew. Sustain. Energy Rev. 2017, vol.71, pp.767-784.

38. Zhang, J.; Pan, G. Comparison and application of multiple regression and BP neural network prediction model. J. Kunming Univ. Sci. Technol. 2013, vol.38, pp.61-67.

39. Zhang, Z.L.; Cheng, X.; Lu, Z.Y.; Gu, D.J. Soc estimation of lithium-ion batteries with aekf and wavelet transform matrix. IEEE Trans. Power Electron. 2017, vol.32, pp.7626-7634.

40. Zou, Y.; Hu, X.; Ma, H.; Li, S.E. Combined state of charge and state of health estimation over lithium-ion battery cell cycle lifespan for electric vehicles. J. Power Sources 2015, vol.273, pp.793-803.

\section{AUTHORS PROFILE}

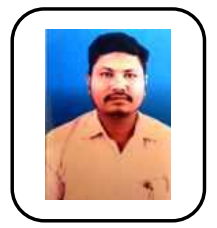

M.Surendar is presently working as a Assistance Professor in Department of Electrical and Electronics Engineering, CSI College of Engineering, Nilgiris, Tamil Nadu. He has obtained B.E Electrical and Electronics Engineering in 2008 at Senguthar Engineering College, Erodeand M.Tech. Power Electronics in PRIST University, Thanjavur in the year of 2012. He has more than eight years teaching experience in CSI College of Engineering, Nilgiris.

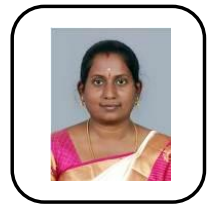

Dr. P. Pradeepa is presently working as a Associate Professor in Department of Electrical and Electronics Engineering at faculty of Engineering and Technology, JAIN Deemed to be University, Bangalore, Karnataka, India. she has more than 15 years teaching experience as a various role in many Engineering Colleges in India and Her research area is Image Processing applications to Bio Medical, Remote Sensing. She has published more than 20 International and National Journals. She has completed B.E. Electrical and Electronics Engineering in 2001 at Government College of Technology, Coimbatore and M.E. Applied Electronics in 2004 at Government College of Engineering, Salem. She has obtained her Ph.D. in 2017 for the field of Information and Communication Engineering from Anna University Chennai. She has membership in various Professional Society such as IAENG, IET, IEEE, ISTE.

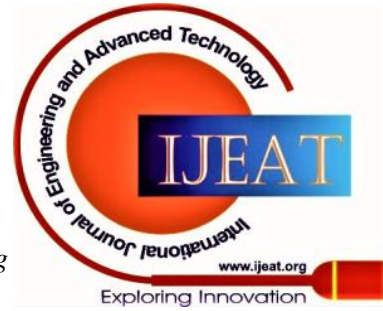

